\title{
INCOMPRESSIBLE FLOW IN ISOTROPIC GRANULAR POROUS MEDIA IN A TIME DEPENDENT DOMAIN
}

\section{João Paulo Lukaszczyk Carlos Antonio Taschetto Leonardo Prange Bonorino}

\begin{abstract}
In this work we study the existence of weak solutions to Navier-Stokes type equations defined in a noncylindrical domain $\hat{\mathrm{Q}}$, where $\hat{\mathrm{Q}}$ is the image of a cylinder $\mathrm{Q}$ of $\mathbb{R}^{n+1}$ and $\hat{\mathrm{Q}}$ is not necessarily increasing or decreasing in time.
\end{abstract}

\section{Introduction}

In this work we will prove results concerning the existence of weak solutions of a system of partial differential equations corresponding to a generalization of the classical Navier-Stokes equations on a noncylindrical domain. The equations are the following:

$$
\left\{\begin{array}{l}
\rho u_{t}+\rho u \cdot \nabla\left(\frac{u}{\eta}\right)-\mu \Delta u+\eta \nabla p+\mu F(\eta) u=\rho \eta f \text { in } \hat{Q}, \\
\operatorname{div} u=0 \text { in } \hat{Q}, \\
u(x, 0)=u_{o}(x), \forall x \in \Omega_{0}, \\
u(x, t)=0, \forall t \in(0, T), \forall x \in \hat{\Sigma} .
\end{array}\right.
$$

Let $T>0$ be a real number and $\left\{\Omega_{t}\right\}, 0 \leq t \leq T$ a family of bounded open sets of $\mathbb{R}^{n}$ with boundary $\partial \Omega_{t}$. Let us consider the noncylindrical domain of $\mathbb{R}^{n+1}$

$$
\hat{Q}=\underset{0<t<T}{\cup} \Omega_{t} \times\{t\} \quad \text { with lateral boundary } \quad \hat{\Sigma}=\underset{0<t<T}{\cup} \partial \Omega_{t} \times\{t\} .
$$

The unknowns in the problem are $u(x, t) \in \mathbb{R}^{n}$ and $p(x, t) \in \mathbb{R}$, which denote, respectively, the fluid velocity and the hydrostatic pressure at a point 
$x \in \Omega$, at time $t \in[0, T]$. We assume that the fluid viscosity, $\mu$, is a positive constant. The density, $\rho$, without loss of generality, will be assumed to be normalized to be one. The porosity $\eta(x, t)$ at a point $x \in \Omega$, at a time $t \in[0, T]$, is defined in rough terms as the void volume divided by the total volume of small regions in the neighborhood of $x$ at a time $t$. Thus, $\eta(x, t)$ assumes real values between zero and one. We observe that the porosity is one in cavities, where, therefore, the flow is free. At points $(x, t)$ such that the porosity is zero, the material medium is purely solid and can be excluded from the flow region. Throughout this work, we will assume that the porosity satisfies $0<\eta(x, t) \leq 1$. $F$ is a force term due to the friction between the granular porous medium and the fluid. On physical grounds, $F$ is a continuous function satisfying $\lim _{z \rightarrow 1} F(z)=0$ and $\lim _{z \rightarrow 0} F(z)=\infty$ (see Prieur du Plessis and Masliyah in [8] for an expression for $F$.) We remark that our results will not depend on that particular expression for $F$. A known external force ficld, such as gravity, is denoted $g(x, t)$ and may be acting on the flow. In cartesian coordinates, we have

$$
\Delta u=\left(\Delta u_{1}, \ldots, \Delta u_{n}\right) \quad \text { and } \quad(u \cdot \nabla u)_{i}=\sum_{j=1}^{n} u_{j} \frac{\partial u_{i}}{\partial x_{j}}
$$

Observe that the classical Navier-Stokes cquations are a particular case of these cquations when $\eta \equiv 1$. There exist several works for Navier-Stokes cquations in noncylindrical domains, among them the works of J.L. Lions [6], R. Salvi [9] and, recently, M. Milla Miranda and J. Límaco Ferrel [7].

This work is organized as follows: for the next section (Preliminaries) we will present the notation, introduce the many functions that will be used through the text. In the third section we establish the transformation between the cylindrical and the noncylindrical problems. In the fourth section we define weak solution and finally in the fifth section we prove our fundamental result of existence of weak solution.

\section{Preliminaries}

Let $\kappa:[0, T] \rightarrow \mathbb{R}^{n^{2}}$ be a function such that $\kappa(t)$ is a $n \times n$ matrix. Let $\Omega$ be an bounded open set of $\mathbb{R}^{n}$ with a smooth boundary $\Gamma$. We can suppose without loss of generality that $0 \in \Omega$. Consider the sets

$$
\Omega_{t}=\{x=\kappa(t) y, y \in \Omega\} ;
$$


where $\kappa(t)=\left(\alpha_{i j}(t)\right)_{n_{\times} n}, y=\left(y_{1}, \ldots, y_{n}\right) \in \Omega \subset \mathbb{R}^{n}$ and

$$
x=\kappa(t) y=\left(\sum_{j=1}^{n} \alpha_{1 j}(t) y_{j}, \ldots, \sum_{j=1}^{n} \alpha_{n j}(t) y_{j}\right) \in \Omega_{t} .
$$

We use the notation $\alpha_{i} \beta_{i}=\sum_{i=1}^{n} \alpha_{i} \beta_{i}$,

$$
\kappa(t)=\left(\alpha_{i j}(t)\right) \quad \text { and } \quad \kappa^{-1}(t)=\left(\beta_{i j}(t)\right),
$$

where $\alpha_{i j}(t)$ are $C^{1}$ functions defined on $[0, T]$ such that det $\kappa(t)>0$. To transform a noncylindrical problem in one defined in a cylindrical domain, we introduce the functions:

$$
\begin{array}{rlrl}
u(x, t) & =v\left(\kappa^{-1}(t) x, t\right), & f(x, t) & =g\left(\kappa^{-1}(t) x, t\right), \\
p(x, t) & =q\left(\kappa^{-1}(t) x, t\right), & u_{0}(x) & =v_{0}\left(\kappa^{-1}(0) x\right), \\
\eta(x, t)=N\left(\kappa^{-1}(t) x, t\right), & F(\eta(x, t)) & =G\left(N\left(\kappa^{-1}(t) x, t\right)\right) .
\end{array}
$$

We introduce the following spaces to obtain the main results:

$$
\nu_{t}=\left\{\varphi \in\left(\mathcal{D}\left(\Omega_{t}\right)\right)^{n} ; \text { div } \varphi=0\right\} \text { and }
$$

$V_{s}\left(\Omega_{t}\right)$ the closure of $\nu_{t}$ with the norm of $\left(H^{s}\left(\Omega_{t}\right)\right)^{n}, s \in \mathbb{R}_{+}$.

In the special cases $s=0$ and $s=1$, we use $V\left(\Omega_{l}\right)=V_{1}\left(\Omega_{l}\right)$ and $H\left(\Omega_{l}\right)=$ $V_{0}\left(\Omega_{t}\right)$. The inner product of $V\left(\Omega_{t}\right), H\left(\Omega_{t}\right)$ and $\left(H^{s}\left(\Omega_{t}\right)\right)^{n}$ are defined by:

$$
(u, z)_{H\left(\Omega_{i}\right)}=\int_{\Omega_{t}} u_{i}(x) z_{i}(x) d x, \quad((u, z))_{V\left(\Omega_{l}\right)}=\int_{\Omega_{t}} \frac{\partial u_{i}(x)}{\partial x_{j}} \frac{\partial z_{i}(x)}{\partial x_{j}} d x
$$

and $((u, z))_{s}=\left(u_{i}, z_{i}\right)_{H^{s}\left(\Omega_{t}\right)}$.

Remark: $\mathrm{V}_{s}\left(\Omega_{t}\right)$ is continuously imbedded in $\left(H_{0}^{1}\left(\Omega_{t}\right)\right)^{n}$ for $s \geq n / 2$, since $s \geq 1, V_{s} \hookrightarrow V \hookrightarrow H \equiv H^{\prime} \hookrightarrow V^{\prime} \hookrightarrow V_{s}^{\prime}$.

In a similar way, we have the spaces over $\Omega$ :

$$
\nu=\left\{\psi \in(\mathcal{D}(\Omega))^{n} ; \operatorname{div}\left(\kappa^{-1}(t) \psi^{l}\right)=0\right\} \text { and }
$$

$V(\Omega)$ the closure of $\nu$ with the norm of $\left(H^{s}(\Omega)\right)^{n}$, 
and, for $s=0$ and $s=1$, we also define $V=V_{1}(\Omega)$ and $H=V_{0}(\Omega)$, provided with the following inner products

$$
(v, w)_{H}=\int_{\Omega} v_{i}(x) w_{i}(x) d x, \quad((v, w))_{V}=\int_{\Omega} \frac{\partial v_{i}(x)}{\partial x_{j}} \frac{\partial w_{i}(x)}{\partial x_{j}} d x
$$

and with the norms $\|v\|_{H}=(v, v)_{H}^{1 / 2}$ and $\|v\|_{V}=((v, v))_{V}^{1 / 2}$. We introduce now the bilinear and trilinear forms corresponding to the variational formulation to the cylindrical and noncylindrical problems.

In the noncylindrical case we define

$$
\begin{gathered}
\hat{a}(t ; u, z)=\int_{\Omega_{t}} \frac{1}{\eta} \frac{\partial u_{i}(x)}{\partial x_{j}} \frac{\partial z_{i}(x)}{\partial x_{j}} d x, \\
\hat{a}_{1}(t ; u, z)=\int_{\Omega_{t}} \frac{\partial u_{i}(x)}{\partial x_{j}} z_{i}(x) \frac{\partial}{\partial x_{j}}\left(\frac{1}{\eta}\right) d x, \\
\hat{b}(t ; u, z, \xi)=\int_{\Omega_{t}} \frac{u_{i}(x)}{\eta} \frac{\partial}{\partial x_{i}}\left(\frac{z_{j}}{\eta}\right) \xi_{j}(x) d x, \\
\hat{d}(t ; u, w)=\int_{\Omega_{+}} F(\eta) u_{i}(x) \frac{w_{i}(x)}{\eta} d x, \\
\hat{e}(t ; u, w)=\int_{\Omega_{i}} u_{i}(x) \frac{\eta^{\prime}}{\eta^{2}} w_{i}(x) d x,
\end{gathered}
$$

and in the cylindrical case

$$
\begin{gathered}
a_{1}(t ; v, w)=\int_{\Omega} \frac{1}{N} \beta_{l j}(t) \beta_{r j}(t) \frac{\partial v_{i}}{\partial y_{r}} \frac{\partial w_{i}}{\partial y_{l}} d y, \\
a_{2}(t ; v, w)=-\int_{\Omega} \beta_{l j}(t) \beta_{r j}(t) \frac{\partial v_{i}}{\partial y_{r}} \frac{\partial N}{\partial y_{l}} \frac{w_{i}}{N^{2}} d y, \\
b_{1}(t ; v, w, \psi)=\int_{\Omega} \frac{v_{i}}{N^{2}} \beta_{l i}(t) \frac{\partial w_{j}}{\partial y_{l}} \psi_{j} d y \\
b_{2}(t ; v, w, \psi)=-\int_{\Omega} \frac{v_{i}}{N^{3}} \beta_{l i}(t) \frac{\partial N}{\partial y_{l}} w_{j} \psi_{j} d y, \\
c(t ; v, w)=\int_{\Omega} \beta_{l r}^{\prime}(t) \alpha_{r j}(t) y_{j} \frac{\partial v_{i}(y)}{\partial y_{l}} \frac{w_{i}(y)}{N} d y,
\end{gathered}
$$




$$
\begin{gathered}
d(t ; v, w)=\int_{\Omega} G(N) v_{i}(y) \frac{w_{i}(y)}{N} d y, \\
e(t ; v, w)=\int_{\Omega} v_{i}(y) \frac{N^{\prime}}{N^{2}} w_{i}(y) d y .
\end{gathered}
$$

Lemma 1 If $\kappa(t)$ satisfies the hypotheses as stated before, then

$$
\frac{(\operatorname{det} \kappa(t))^{\prime}}{\operatorname{det} \kappa(t)}=-\operatorname{tr}\left(\left(\kappa^{-1}(t)\right)^{\prime} \kappa(t)\right) \text {. }
$$

\section{The Equation in Q}

If $x \in \Omega_{t}$ and $y \in \Omega$ satisfy (1), using (2) we have

$$
\begin{gathered}
x_{r}=\alpha_{r j}(t) y_{j} e y_{l}=\beta_{l r}(t) x_{r} \\
\frac{\partial y_{l}}{\partial t}=\beta_{l r}^{\prime}(t) x_{r}=\beta_{l r}^{\prime}(t) \alpha_{r j}(t) y_{j} \\
\frac{\partial y_{l}}{\partial x_{j}}=\beta_{l j}(t) .
\end{gathered}
$$

From $(3), u_{i}(x, t)=v_{i}(y, t)$, we get

$$
\frac{\partial u_{i}(x, t)}{\partial x_{j}}=\frac{\partial v_{i}(y, t)}{\partial y_{l}} \frac{\partial y_{l}}{\partial x_{j}}
$$

and using (20) we obtain

$$
\frac{\partial u_{i}(x, t)}{\partial x_{j}}=\beta_{l j}(t) \frac{\partial v_{i}(y, t)}{\partial y_{l}},
$$

For $j$ fixed, we also have

$$
\begin{gathered}
\frac{\partial^{2} u_{i}(x, t)}{\partial x_{j}^{2}}=\frac{\partial}{\partial x_{j}}\left(\beta_{l j}(t) \frac{\partial v_{i}(y, t)}{\partial y_{l}}\right)=\beta_{l j}(t) \frac{\partial}{\partial x_{j}}\left(\frac{\partial v_{i}(y, t)}{\partial y_{l}}\right), \\
\frac{\partial^{2} u_{i}(x, t)}{\partial x_{j}^{2}}=\beta_{l j}(t) \frac{\partial^{2} v_{i}(y, t)}{\partial y_{r} \partial y_{l}} \frac{\partial y_{r}}{\partial x_{j}}
\end{gathered}
$$

so

$$
\Delta u_{i}(x, t)=\beta_{l j}(t) \beta_{r, j}(t) \frac{\partial^{2} v_{i}(y, t)}{\partial y_{r} \partial y_{l}}
$$


On the other hand,

$$
\begin{aligned}
& u_{i}(x, t) \frac{\partial}{\partial x_{i}}\left(\frac{u}{\eta}\right)(x, t)=\frac{u_{i}(x, t)}{\eta} \frac{\partial u}{\partial x_{i}}(x, t)+u_{i}(x, t) \frac{\partial}{\partial x_{i}}\left(\frac{1}{\eta}\right) u(x, t)= \\
& =\frac{u_{i}(x, t)}{\eta} \frac{\partial u}{\partial x_{i}}(x, t)-\frac{u_{i}(x, t)}{\eta^{2}} \frac{\partial \eta}{\partial x_{i}} u(x, t)
\end{aligned}
$$

Then

$$
u_{i}(x, t) \frac{\partial}{\partial x_{i}}\left(\frac{u}{\eta}\right)(x, t)=\frac{u_{i}(x, t)}{\eta} \frac{\partial u_{j}}{\partial x_{i}}(x, t)-\frac{u_{i}(x, t)}{\eta^{2}} \frac{\partial \eta}{\partial x_{i}} u_{j}(x, t)
$$

for $j=1,2, \ldots, n$ and using (22)

$$
u_{i}(x, t) \frac{\partial}{\partial x_{i}}\left(\frac{u}{\eta}\right)(x, t)=\frac{v_{i}(y, t)}{N} \beta_{l i}(t) \frac{\partial v_{j}(y, t)}{\partial y_{l}}-\frac{v_{j}(y, t)}{N^{2}} \beta_{l i}(t) \frac{\partial N}{\partial y_{l}} v_{j}(y, t) .
$$

Since $\frac{\partial u_{i}(x, t)}{\partial t}=\frac{\partial v_{i}(y, t)}{\partial y_{l}} \frac{\partial y_{l}}{\partial t}+\frac{\partial v_{i}(y, t)}{\partial t}$, using (19) we get

$$
\frac{\partial u_{i}(x, t)}{\partial t}=\beta_{l r}^{\prime}(t) \alpha_{r j}(t) y_{j} \frac{\partial v_{i}(y, t)}{\partial y_{l}}+\frac{\partial v_{i}(y, t)}{\partial t} .
$$

From (4) we have $\frac{\partial p(x, t)}{\partial x_{i}}=\frac{\partial q(y, t)}{\partial y_{l}} \frac{\partial y_{l}}{\partial x_{i}}=\beta_{l i}(t) \frac{\partial q(y, t)}{\partial y_{l}}$, hence and by (20),

$$
\frac{\partial p(x, t)}{\partial x_{i}}=\left(\frac{\partial q(y, t)}{\partial y_{1}}, \ldots, \frac{\partial q(y, t)}{\partial y_{n}}\right)\left(\beta_{1 i}(t), \ldots, \beta_{n i}(t)\right)=\left(\nabla q . \kappa^{-1}(t)\right)_{i},
$$

that is $\quad \nabla p(x, t)=\nabla q(y, t) \cdot \kappa^{-1}(t)$.

For each $i$ fixed, from (22), the following relations hold:

$$
\frac{\partial u_{i}(x, t)}{\partial x_{i}}=\sum_{l=1}^{n} \beta_{l i}(t) \frac{\partial v_{i}(y, t)}{\partial y_{l}} \text { and div } u(x, t)=\sum_{i} \frac{\partial u_{i}(x, t)}{\partial x_{i}}
$$

Therefore div $u(x, t)=\beta_{l i}(t) \frac{\partial v_{i}(y, t)}{\partial y_{l}}$.

So

$$
\begin{gathered}
\text { div } u(x, t)=\left[\frac{\partial}{\partial y_{1}}\left(\beta_{1 i} v_{i}(y, t)\right)+\ldots+\frac{\partial}{\partial y_{n}}\left(\beta_{n i} v_{i}(y, t)\right)\right], \\
\operatorname{div} u(x, t)=\left[\left(\frac{\partial}{\partial y_{1}}, \ldots, \frac{\partial}{\partial y_{n}}\right)\left(\kappa^{-1}(t) \cdot v^{l}(y, t)\right)\right]=\nabla \cdot\left(\kappa^{-1}(t) \cdot v^{l}(y, t)\right),
\end{gathered}
$$

therefore

$$
\operatorname{div} u(x, t)=\operatorname{div}\left(\kappa^{-1}(t) \cdot v^{t}(y, t)\right) .
$$


From (5) $\frac{\partial \eta}{\partial x_{j}}=\frac{\partial N}{\partial y_{l}} \frac{\partial y_{l}}{\partial x_{j}}$, by $(20)$, we have

$$
\frac{\partial \eta}{\partial x_{j}}=\beta_{l j}(t) \frac{\partial N}{\partial y_{l}} .
$$

On the other hand, $\frac{\partial \eta}{\partial t}=\frac{\partial N}{\partial y_{l}} \frac{\partial y_{l}}{\partial t}+\frac{\partial N}{\partial t}$, using (19) we get

$$
\frac{\partial \eta}{\partial t}=\beta_{l t}^{\prime}(t) \alpha_{r j}(t) y_{j} \frac{\partial N}{\partial y_{l}}+\frac{\partial N}{\partial t}
$$

Replacing (23) to (30) in (PNC), we conclude for $i=1, \ldots, n$

$$
\begin{gathered}
\beta_{l r}^{\prime}(t) \alpha_{r j}(t) y_{j} \frac{\partial v_{i}(y, t)}{\partial y_{l}}+\frac{\partial v_{i}(y, t)}{\partial t}-\mu \beta_{l j}(t) \beta_{r j}(t) \frac{\partial^{2} v_{i}(y, t)}{\partial y_{r} \partial y_{l}}+ \\
+\frac{v_{j}(y, t)}{N} \beta_{l j}(t) \frac{\partial v_{i}(y, t)}{\partial y_{l}}-\frac{v_{j}(y, t)}{N^{2}} \beta_{l j}(t) \frac{\partial N}{\partial y_{l}} y_{i}(y, t)+ \\
+N(y, t) \frac{\partial q(y, t) \cdot \kappa^{-1}(t)}{\partial y_{l}}+G(N(y, t)) v_{i}(y, t)=N(y, t) g_{i}(y, t) \text { in } Q .
\end{gathered}
$$

So

$$
\left\{\begin{array}{l}
v^{\prime}-\mu \frac{\partial}{\partial y_{l}}\left(\beta_{l j}(t) \beta_{r j}(t) \frac{\partial v(y, t)}{\partial y_{r}}\right)+\frac{v_{j}(y, t)}{N} \beta_{l j}(t) \frac{\partial v(y, t)}{\partial y_{l}} \\
-\frac{v_{j}(y, t)}{N^{2}} \beta_{l j}(t) \frac{\partial N}{\partial y_{l}} v(y, t)+\beta_{l r}^{\prime}(t) \alpha_{r j}(t) y_{j} \frac{\partial v(y, t)}{\partial y_{l}}+ \\
N(y, t) \nabla q(y, t) . \kappa^{-1}(t)+\mu G(N(y, t)) v(y, t)=N(y, t) g(y, t) \text { in } Q \\
\operatorname{div}\left[\kappa^{-1}(t) \cdot v^{t}(y, t)\right]=0 \text { in } Q \\
v=0 \text { in } \Sigma \\
v(y, 0)=v_{0}(y) \text { with } y \in \Omega .
\end{array}\right.
$$

\section{Definition of Weak Solution}

Definition 1 Notion of weak solution to the problem (PNC).

To define the weak solution of (PNC) we will climinate the pressure as in classical Navier-Stokes equations. For that, we make the inner product in $L^{2}(\Omega)$ of the equation (divided by $\eta$ ) by a function of $V$ and observing that

$$
\frac{d}{d t}\left(\frac{u}{\eta}\right)=\frac{u^{\prime}}{\eta}-\frac{u \eta^{\prime}}{\eta^{2}}
$$


we can rewrite (PNC) in the following way:

For $f, \eta$ and $u_{0}$ given, with $f \in L^{2}\left(0, T, V\left(\Omega_{t}\right)^{\prime}\right)$ and $u_{0} \in H\left(\Omega_{t}\right)$ we have to find $u$ satisfying $u \in L^{2}\left(0, T, V\left(\Omega_{t}\right)\right)$ and:

$$
\left\{\begin{array}{l}
-\left(u, \frac{\xi^{\prime}}{\eta}\right)+\left(\frac{\eta^{\prime}}{\eta^{2}} u, \xi\right)+\left(\frac{u}{\eta} \cdot \nabla\left(\frac{u}{\eta}\right), \xi\right)+\mu\left(\nabla(u), \nabla\left(\frac{1}{\eta}\right) \xi\right) \\
+\mu\left(\nabla(u), \nabla(\xi) \frac{1}{\eta}\right)+\mu\left(\frac{F(\eta)}{\eta} u, \xi\right)=\langle f, \xi\rangle \quad \forall \xi \in V \text { in } \mathcal{D}^{\prime}(0, T) \\
u(0)=u_{0} \in H .
\end{array}\right.
$$

Integrating in $[0, T]$, and making use of $(6)$ and $(10)$, we obtain

$$
\left\{\begin{array}{l}
u \in L^{2}\left(0, T, V\left(\Omega_{t}\right)\right) \cap L^{\infty}\left(0, T, H\left(\Omega_{t}\right)\right) \\
-\int_{0}^{T}\left(u, \xi^{\prime} / N\right)_{H\left(\Omega_{t}\right)} d t+\mu \int_{0}^{T} \hat{a}(t ; u, \xi) d t+\mu \int_{0}^{T} \hat{a}_{1}(t ; u, \xi) d t+ \\
\int_{0}^{T} \hat{b}(t ; u, u, \xi) d t+\int_{0}^{T} \hat{d}(t ; u, \xi) d t+\int_{0}^{T} \hat{e}(t ; u, \xi) d t=\int_{0}^{T}(f, \xi)_{I\left(\Omega_{t}\right)} d t \\
\forall \xi \in L^{2}\left(0, T ; V\left(\Omega_{l}\right) \cap L^{n}\left(\Omega_{l}\right)^{n}\right), \xi^{\prime} \in L^{2}\left(0, T ; H\left(\Omega_{l}\right)\right) \\
\xi(0)=0, \xi(T)=0 \\
u(0)=u_{0}
\end{array}\right.
$$

(PNC1)

Definition 2 Notion of weak solution to the problem $(P C)$.

In this case we divide (PC) by $N$, then multiply by a suitable function $\psi$ and integrate in $\Omega$, obtaining

$$
\begin{gathered}
\int_{\Omega} \frac{v^{\prime}}{N} \psi d y-\int_{\Omega} \frac{\mu}{N} \frac{\partial}{\partial y_{l}}\left(\beta_{l j}(t) \beta_{r j}(t) \frac{\partial v}{\partial y_{l}}\right) \psi d y+\int_{\Omega} \frac{v_{j}(y, t)}{N^{2}} \beta_{l j}(t) \frac{\partial v(y, t)}{\partial y_{l}} \psi d y \\
\quad-\int_{\Omega} \frac{v_{j}(y, t)}{N^{3}} \beta_{l j}(t) \frac{\partial N}{\partial y_{l}} v(y, t) \psi d y+\int_{\Omega} \frac{1}{N} \beta_{l r}^{\prime}(t) \alpha_{r j}(t) y_{j} \frac{\partial v}{\partial y_{l}} \psi d y+ \\
+\int_{\Omega} \nabla q(y, t) \kappa^{-1}(t) \psi d y+\int_{\Omega} \frac{\mu G(N)}{N} v \psi d y=\int_{\Omega} g \psi d y .
\end{gathered}
$$

Making use of (11) to (17), we have

$$
\begin{aligned}
& \int_{\Omega} \frac{v^{\prime}}{N} \cdot \psi d y=\int_{\Omega}\left(\left(\frac{v^{\prime}}{N}-\frac{v N^{\prime}}{N^{2}}\right) \cdot \psi+\frac{v N^{\prime}}{N^{2}} \psi\right) d y=-\left(\frac{v}{N}, \psi^{\prime}\right)+e(t ; v, \psi) \\
& \int_{\Omega} \frac{1}{N} \frac{\partial}{\partial y_{l}}\left(\beta_{l j}(t) \beta_{r j}(t) \frac{\partial v}{\partial y_{l}}\right) \psi d y=\int_{\Omega} \beta_{l j}(t) \beta_{r j}(t) \frac{\partial v_{i}}{\partial y_{r}} \frac{\partial}{\partial y_{l}}\left(\frac{\psi_{i}}{N}\right) d y= \\
& \int_{\Omega} \frac{1}{N} \beta_{l j}(t) \beta_{r j}(t) \frac{\partial v_{i}}{\partial y_{r}} \frac{\partial \psi_{i}}{\partial y_{l}} d y-\int_{\Omega} \beta_{l j}(t) \beta_{r j}(t) \frac{\partial v_{i}}{\partial y_{r}} \frac{\partial N}{\partial y_{l}} \frac{\psi_{i}}{N^{2}} d y= \\
& =a_{1}(t ; v, \psi)+a_{2}(t ; v, \psi)
\end{aligned}
$$




$$
\begin{gathered}
\int_{\Omega} \frac{1}{N} \beta_{l i}(t) v_{i} \frac{\partial}{\partial y_{l}}\left(\frac{v}{N}\right) \psi d y=\int_{\Omega} \beta_{l i}(t) v_{i} \frac{\partial v_{j}}{\partial y_{l}} \frac{\psi_{j}}{N^{2}} d y- \\
\int_{\Omega} \beta_{l i}(t) \frac{v_{i}}{N^{3}} \frac{\partial y_{l}}{\partial y_{l}} v_{j} \psi_{j} d y=b_{1}(t ; v, v, \psi)+b_{2}(t ; v, v, \psi) \\
\int_{\Omega} \frac{1}{N} \beta_{l r}^{\prime}(t) \alpha_{r j}(t) y_{j} \frac{\partial v}{\partial y_{l}} \psi d y=\int_{\Omega} \beta_{l r}^{\prime}(t) \alpha_{r j}(t) y_{j} \frac{\partial v_{i}}{\partial y_{l}} \frac{\psi_{i}}{N} d y=c(t ; v, \psi) \\
\int_{\Omega} \nabla q \kappa^{-1}(t) . \psi d y=\int_{\Omega} q \cdot \operatorname{div}\left(\kappa^{-1}(t) \psi^{t}\right) d y=0 .
\end{gathered}
$$

$\int_{\Omega} \frac{G(N)}{N} v \psi d y=\int_{\Omega} G(N) v_{i} \frac{\psi_{i}}{N} d y=d(t ; v, \psi)$ and $\int_{\Omega} g \cdot \psi d y=\int_{\Omega} g_{i} \cdot \psi_{i} d y=(g, \psi)$

so

$$
\left\{\begin{array}{l}
v \in L^{2}(0, T ; V) \cap L^{\infty}(0, T ; H) \\
-\int_{0}^{T}\left(\frac{v}{N}, \psi^{\prime}\right) d t+\mu \int_{0}^{T} a(t ; v, \psi) d t+\int_{0}^{T} b_{1}(t ; v, v, \psi) d t+\int_{0}^{T} b_{2}(t ; v, v, \psi) d t \\
+\int_{0}^{T} c(t ; v, \psi) d t+\mu \int_{0}^{T} d(t ; v, \psi) d t+\int_{0}^{T} e(t ; v, \psi) d t=\int_{0}^{T}(g, \psi) d t \\
\forall \psi \in L^{2}\left(0, T ; V \cap L^{n}(\Omega)^{n}\right), \psi^{t} \in L^{2}(0, T ; H) \\
\psi(0)=0, \psi(T)=0, \quad v(0)=v_{0}
\end{array}\right.
$$

Theorem 1 Problems (PNC1) and (PC1) are equivalent.

Proof. Recalling from (1),(2) and (3) we have

$$
x=\kappa(t) y, \quad y=\kappa^{-1}(t) x, \quad x_{r}=\alpha_{r j} y_{j}, \quad y_{l}=\beta_{l r} x_{r},
$$

we have established that $u(x, t)=v\left(\kappa^{-1}(t) x, t\right)$.

Let $\xi(x, t)=\left|\operatorname{det} \kappa^{-1}(t)\right| \psi\left(\kappa^{-1}(t) x, t\right)$. Then

$$
\begin{gathered}
\frac{\partial \xi_{i}(x, t)}{\partial t}=\left|\operatorname{det} \kappa^{-1}(t)\right|\left(\frac{\partial \psi_{i}(y, t)}{\partial y_{l}} \frac{\partial y_{l}}{\partial t}+\frac{\partial \psi_{i}(y, t)}{\partial t}\right)+\left|\operatorname{det} \kappa^{-1}(t)\right|^{\prime} \psi_{i}(y, t) \\
\frac{\partial \xi_{i}(x, t)}{\partial t}=\left|\operatorname{det}^{-1}(t)\right|\left(\frac{\partial \psi_{i}}{\partial y_{l}} \beta_{l r}^{\prime} \alpha_{r j} y_{j}+\frac{\partial \psi_{i}}{\partial t}\right)+\left|\operatorname{det} \kappa^{-1}(t)\right|^{\prime} \psi_{i}
\end{gathered}
$$


Now we compute each integral in $\Omega_{t}$ of problem (PNC1) and making use of (32) we have

$$
\left(u, \frac{\xi^{\prime}}{\eta}\right)_{H\left(\Omega_{t}\right)}=\int_{\Omega}\left(\frac{v_{i}}{N} \frac{\partial \psi_{i}}{\partial y_{l}} \beta_{l r}^{\prime} \alpha_{r j} y_{j}+\frac{v_{i}}{N} \frac{\partial \psi_{i}}{\partial t}-\frac{1}{N} \frac{(\operatorname{det} \kappa(t))^{\prime}}{\operatorname{det} \kappa(t)} v_{i} \psi_{i}\right) d y\left|\operatorname{det} \kappa^{-1}(t)\right|
$$

On the other hand $\beta_{l r}^{\prime} \alpha_{r l}=t_{r}\left(\left(\kappa^{-1}(t)\right)^{\prime} \kappa(t)\right)$ then, by lemma 1

$$
\beta_{l r}^{\prime} \alpha_{r l}=-\frac{(\operatorname{det} \kappa(t))^{\prime}}{\operatorname{det} \kappa(t)}
$$

where $t_{r}(A)$ denotes the trace of the $n \times n$ matrix $A$.

Let $F$ be the following vector field $\left(0, \ldots, \frac{v_{i} \psi_{i}}{N} \beta_{l r}^{\prime} \alpha_{r j} y_{j}, 0, \ldots, 0\right)$ where the non null component ocupies the $l$-position, then

$$
\begin{aligned}
\operatorname{div} F & =\frac{\partial}{\partial y_{l}}\left(\frac{v_{i} \psi_{i}}{N}\left[\beta_{l r}^{\prime} \alpha_{r j} y_{j}\right]\right)=\frac{\partial}{\partial y_{l}}\left(\frac{v_{i} \psi_{i}}{N}\right) \cdot\left[\beta_{l r}^{\prime} \alpha_{r j} y_{j}\right]+\frac{v_{i} \psi_{i}}{N} \frac{\partial}{\partial y_{l}}\left(\beta_{l r}^{\prime} \alpha_{r j} y_{j}\right)= \\
& =\frac{1}{N} \frac{\partial v_{i}}{\partial y_{l}} \psi_{i} \beta_{l r}^{\prime} \alpha_{r j} y_{j}+v_{i} \frac{\partial}{\partial y_{l}}\left(\frac{\psi_{i}}{N}\right) \beta_{l r}^{\prime} \alpha_{r j} y_{j}+\frac{v_{i} \psi_{i}}{N} \beta_{l r}^{\prime} \frac{\partial}{\partial y_{l}}\left(\alpha_{r j} y_{j}\right),
\end{aligned}
$$

but

$$
\frac{\partial}{\partial y_{l}}\left(\alpha_{r j} y_{j}\right)=\frac{\partial}{\partial y_{l}}\left(\alpha_{r 1} y_{1}+\ldots+\alpha_{r n} y_{n}\right)=\alpha_{r l}
$$

then

$$
\operatorname{div} F=\frac{1}{N} \frac{\partial v_{i}}{\partial y_{l}} \psi_{i} \beta_{l r}^{\prime} \alpha_{r j} y_{j}+v_{i} \frac{\partial}{\partial y_{l}}\left(\frac{\psi_{i}}{N}\right) \beta_{l r}^{\prime} \alpha_{r j} y_{j}+\frac{v_{i} \psi_{i}}{N} \beta_{l r}^{\prime} \alpha_{r l},
$$

and making use of (34)

$$
\operatorname{div} F=\frac{1}{N} \frac{\partial v_{i}}{\partial y_{l}} \psi_{i} \beta_{l r}^{\prime} \alpha_{r j} y_{j}+v_{i} \frac{\partial}{\partial y_{l}}\left(\frac{\psi_{i}}{N}\right) \beta_{l r}^{\prime} \alpha_{r j} y_{j}-\frac{1}{N} \frac{(\operatorname{det} \kappa(t))^{\prime}}{\operatorname{det} \kappa(t)} v_{i} \psi_{i} .
$$

From Green's Theorem, $\int_{\Omega} d i v F d y=\int_{\hat{\Sigma}} F \cdot \vec{\eta} d s=0$, then

$$
\int_{\Omega}\left(\frac{1}{N} \frac{\partial v_{i}}{\partial y_{l}} \psi_{i} \beta_{l r}^{\prime} \alpha_{r j} y_{j}+v_{i} \frac{\partial}{\partial y_{l}}\left(\frac{\psi_{i}}{N}\right) \beta_{l r}^{\prime} \alpha_{r j} y_{j}-\frac{1}{N} \frac{(\operatorname{det} \kappa(t))^{\prime}}{\operatorname{det} \kappa(t)} v_{i} \psi_{i}\right) d y=0
$$

and

$$
\begin{aligned}
& -\int_{\Omega}\left(\frac{v_{i}}{N} \frac{\partial \psi_{i}}{\partial y_{l}} \beta_{l r}^{\prime} \alpha_{r j} y_{j}-\frac{1}{N} \frac{(\operatorname{det} \kappa(t))^{\prime}}{\operatorname{det} \kappa(t)} v_{i} \psi_{i}\right) d y= \\
& =\int_{\Omega}\left(v_{i} \psi_{i} \frac{\partial}{\partial y_{l}}\left(\frac{1}{N}\right) \beta_{l r}^{\prime} \alpha_{r j} y_{j}+\frac{\beta_{l r}^{\prime} \alpha_{r j}}{N} y_{j} \frac{\partial v_{i}}{\partial y_{l}} \psi_{i}\right) d y,
\end{aligned}
$$


combining (35) and (33), we get

$$
\begin{gathered}
\left(u, \frac{\xi^{\prime}}{\eta}\right)_{H\left(\Omega_{t}\right)}=\int_{\Omega} \frac{v_{i}}{N} \frac{\partial \psi_{i}}{\partial t}-\frac{\beta_{l r}^{\prime} \alpha_{r j}}{N} y_{j} \frac{\partial v_{i}}{\partial y_{l}} \psi_{i}-v_{i} \psi_{i} \frac{\partial}{\partial y_{l}}\left(\frac{1}{N}\right) \beta_{l r}^{\prime} \alpha_{r j} y_{j} d y\left|\operatorname{det} \kappa^{-1}(t)\right| \\
-\left(u, \frac{\xi^{\prime}}{\eta}\right)_{H\left(\Omega_{l}\right)}=\left[-\left(v, \frac{\psi^{\prime}}{N}\right)+c(t ; v, \psi)+c_{1}(t ; v, \psi)\right]\left|\operatorname{det} \kappa^{-1}(t)\right|
\end{gathered}
$$

where

$$
\int_{\Omega} v_{i} \psi_{i} \frac{\partial}{\partial y_{l}}\left(\frac{1}{N}\right) \beta_{l r}^{\prime} \alpha_{r j} y_{j} d y\left|\operatorname{det} \kappa^{-1}(t)\right|=c_{1}(t ; v, \psi) .
$$

From (6) and making use of (22) we have

$$
\hat{a}(t ; u, \xi)=\left[\int_{\Omega} \frac{\beta_{l j} \beta_{r j}}{N} \frac{\partial v_{i}}{\partial y_{l}} \frac{\partial \psi_{i}}{\partial y_{r}} d y\right]\left|\operatorname{det} \kappa^{-1}(t)\right|=a_{1}(t ; v, \psi)\left|\operatorname{det} \kappa^{-1}(t)\right|,
$$

but

$$
\begin{aligned}
& \hat{a}_{1}(t ; u, \xi)=\int_{\Omega_{t}} \frac{\partial u_{i}(x)}{\partial x_{j}} \xi_{i}(x) \frac{\partial}{\partial x_{j}}\left(\frac{1}{\eta}\right) d x= \\
& =-\int_{\Omega} \frac{1}{N^{2}} \beta_{l j}(t) \beta_{r j}(t) \frac{\partial v_{i}(y)}{\partial y_{l}} \frac{\partial N}{\partial y_{r}} \psi_{i}(y) d y\left|\operatorname{det} \kappa^{-1}(t)\right|=a_{2}(t ; v, \psi)\left|\operatorname{det} \kappa^{-1}(t)\right| .
\end{aligned}
$$

From $(8), \hat{b}(t ; u, u, \xi)=$

$$
=\left[\int_{\Omega} \frac{v_{i}}{N^{2}} \beta_{l j}(t) \frac{\partial v_{j}}{\partial y_{l}} \psi_{j}(y) d y-\int_{\Omega} \frac{v_{i}}{N^{3}} \beta_{l j}(t) v_{j} \frac{\partial N}{\partial y_{l}} \psi_{j}(y) d y\right]\left|\operatorname{det} \kappa^{-1}(t)\right|
$$

then by (13) and (14), we have

$$
\hat{b}(t ; u, u, \xi)=\left(b_{1}(t ; v, v, \psi)+b_{2}(t ; v, v, \psi)\right)\left|\operatorname{det} \kappa^{-1}(t)\right| .
$$

From (9)

$$
\hat{d}(t ; u, \xi)=\int_{\Omega} G(N) v_{i}(y) \frac{\psi_{i}(y)}{N} d y\left|\operatorname{det} \kappa^{-1}(t)\right|=d(t ; v ; \psi)\left|\operatorname{det} \kappa^{-1}(t)\right| .
$$

We also have from (10)

$$
\begin{aligned}
& \hat{e}(t ; u, \xi)=\left[\int_{\Omega} \frac{v_{i}(y)}{N^{2}} \beta_{l r}^{\prime}(t) \alpha_{r j} y_{j} \frac{\partial N}{\partial y_{l}} \psi_{i}(y) d y+\int_{\Omega} \frac{v_{i}(y)}{N^{2}} \frac{\partial N}{\partial t} \psi_{i}(y) d y\right]\left|\operatorname{det} \kappa^{-1}(t)\right| \\
& =\left[-c_{1}(t ; v, \psi)+e(t ; v, \psi)\right]\left|\operatorname{det} \kappa^{-1}(t)\right|
\end{aligned}
$$


Finally, integrating both sides of expressions (36) to (41) and adding term by term we conclude that problems (PNC1) and (PC1) are equivalent.

Define the forms concerning to the cylindrical problem:

$$
\begin{gathered}
a(t ; v, w)=a_{1}(t ; v, w)+a_{2}(t ; v, w)=\int_{\Omega} \beta_{l j}(t) \beta_{r j}(t) \frac{\partial v i}{\partial y_{r}} \frac{\partial}{\partial y_{l}}\left(\frac{w_{i}}{N}\right) d y . \\
b(t ; v, w, \psi)=b_{1}(t ; v, w, \psi)+b_{2}(t ; v, w, \psi)=\int_{\Omega} \beta_{l i}(t) v_{i} \frac{\partial}{\partial y_{l}}\left(\frac{w_{j}}{N}\right) \frac{\psi_{j}}{N} d y .
\end{gathered}
$$

Definition 3 Let $A(t):\left(H_{0}^{1}(\Omega)\right)^{n} \rightarrow\left(H^{-1}(\Omega)\right)^{n}$ be the operator defined by

$$
A(t) v=-\frac{1}{N} \frac{\partial}{\partial y_{l}}\left(\beta_{l j}(t) \beta_{r j}(t) \frac{\partial v(y, t)}{\partial y_{r}}\right) \text { for } v \in\left(H_{0}^{1}(\Omega)\right)^{n},
$$

Lemma 2 The linear form $a(t ; v, w)$ defined in (42) and the operator $A(t)$ defined in (44) satisfy

$$
\begin{array}{lll}
i . & \langle A(t) v, w\rangle=a(t ; v, w), \quad \forall v, w \in V \\
i i . & |a(t ; v, w)| \leq C\|v\|\|w\|, \quad \forall v, w \in V .
\end{array}
$$

Lemma 3 The linear form $a_{1}(t ; v, w)$ as defined in (11) is coercive and continuous, that is,

i. $a_{1}(t ; v, v) \geq a_{0}\|v\|^{2}, \forall v \in V$, where $a_{0}>0$

ii. $a_{1}(t ; v, w) \leq C\|v\|\|w\|, \forall v, w \in V$.

Lemma 4 Let $b(t ; v, w, \psi), c(t ; v, w), d(t ; v, w)$ and $e(t ; v, w)$ the multilinear forms defined by (43), (15), (16) and (17) respectively. Then

i. $|b(t ; v, w, \psi)| \leq C\|v\|\|w\|\|\psi\|_{V \cap\left(L^{n}(\Omega)\right)^{n}} \quad \forall v, w \in V$ and $\psi \in V \cap\left(L^{n}(\Omega)\right)^{n}$.

ii. $b(t ; v, v, w)=-b(t ; v, w, v) \quad \forall v \in V$ and $w \in V_{s}(\Omega)$, where $s=\frac{n}{2}$.

iii. For $v \in V$, the linear form $w \mapsto b(t ; v, v, w)$ is continuous on $V_{s}(\Omega)\left(s=\frac{n}{2}\right)$ and $b(t ; v, v, w)=\langle B(t) v, w\rangle_{V_{s}^{\prime} V_{s}}$, where $B(t) v \in V_{s}^{\prime}(\Omega)$ and

$$
\|B(t) v\|_{V_{s}^{\prime}} \leq C\|v\|_{\left(L^{p}(\Omega)\right)}^{2} \quad \text { with } \frac{1}{p}=\frac{1}{2}-\frac{1}{2 n} .
$$

iv. $|c(t ; v, w)| \leq C\|v\|\|w\| \quad \forall v, w \in H$.

$v$. For $v \in V$, the linear form $w \mapsto c(t ; v, w)$ is continuous on $H$ and

$$
c(t ; v, w)=\langle C(t) v, w\rangle_{H^{\prime} H}=(C(t) v, w),
$$


where $C(t) v \in H^{\prime}=H$ and $|C(t) v| \leq C\|v\|$.

vi. For $v \in V$, the linear form $w \mapsto d(t ; v, w)$ is continuous on $H$,

$$
d(t ; v, w)=\langle D(t) v, w\rangle_{H^{\prime} H}=(D(t) v, w),
$$

and $|D(t) v| \leq C\|v\|$.

vii. For $v \in V$, the linear form $w \mapsto e(t ; v, w)$ is continuous on $H$,

$$
e(t ; v, w)=\langle E(t) v, w\rangle_{I^{\prime} I}=(E(t) v, w)
$$

and $|E(t) v| \leq C\|v\|$.

viii. $L^{2}(0, T ; V) \cap L^{\infty}(0, T ; H) \subset L^{4}\left(0, T ;\left(L^{p}(\Omega)\right)^{n}\right)$, where $p$ is given by (45).

The three last lemmas can be found in MIRANDA [7], pg 253-254.

\section{Existence of Solution}

In this section we will prove the following result

Theorem 2 (existence of weak solutions)

Let $\Omega \subset \mathbb{R}^{n}$, with $n=2,3$, be an open bounded set with regular boundary and $T>0$. Also, let be given, $u_{0} \in H\left(\Omega_{T}\right), g \in L^{2}\left(0, T, V_{s}^{\prime}\left(\Omega_{T}\right)\right)$ and a continuous function $F:(0,1] \rightarrow \mathbb{R}^{+}$. Suppose that the porosity $n: \hat{Q} \rightarrow(0,1]$ satisfies

$$
\begin{aligned}
& 0<n_{0} \leq n(x, t)<1 \quad \forall(x, t) \in \hat{Q} \\
& n^{\prime} \in L^{2}\left(0, T, L^{\frac{3}{2}}\left(\Omega_{T}\right)\right) \cap L^{1}\left(0, T, L^{\infty}\left(\Omega_{T}\right)\right) \\
& \nabla n \in L^{2}\left(0, T, L^{\infty}\left(\Omega_{T}\right)\right) \cap L^{\infty}\left(0, T, L^{3}\left(\Omega_{T}\right)\right)
\end{aligned}
$$

Then, there exists a solution $u \in L^{2}\left(0, T, V\left(\Omega_{T}\right)\right) \cap L^{\infty}\left(0, T, H\left(\Omega_{T}\right)\right)$ of (PNC1).

Proof. For $s=n / 2$ the injection $V_{s} \hookrightarrow H$ is compact, since $V_{s} \subset V_{1} \equiv V \hookrightarrow H$ and $H_{0}^{1}(\Omega)$ is compactly imbedded in $L^{2}(\Omega)$ (see Lions [6], pg 66).

This result guarantees the existence of solution to the spectral problem

$$
((w, v))_{H^{s}(\Omega)}=\lambda(w, v) \quad \forall v \in V_{s}(\Omega) .
$$

Consider an orthonormal basis of $V_{s}(\Omega)$ generated by a countable set of eigenvectors $\left(w_{n}\right)$ corresponding to the set of positive eigenvalues $\left(\lambda_{\mu}\right)$. We will use $\left(w_{n}\right)$ in Galerkin's methods. For each $m$ we define an approximate solution $v_{m}$ 
to the problem ( $\mathrm{PC} 1)$. Let $V_{m}$ be the subspaces generated by the first vectors $w_{1}, \ldots, w_{m}$, that is, if $v_{m}(t) \in V_{m}$, then

$$
v_{m}(t, y)=\sum_{j=1}^{m} h_{j m}(t) u_{j}(y)
$$

where $h_{j m}$ are scalar functions defined in $[0, T]$. Consider the approximate problem

$$
\left\{\begin{array}{l}
\left(\frac{v_{m}^{\prime}(t)}{N}, w_{j}\right)+\mu a\left(t ; v_{m}(t), w_{j}\right)+b\left(t ; v_{m}(t), v_{m}(t), w_{j}\right)+c\left(t ; v_{m}(t), w_{j}\right) \\
+\mu d\left(t ; v_{m}(t), w_{j}\right)=\left(g, w_{j}\right) \quad j=1, \ldots, m \text { and } v_{m}(0)=v_{0 m}, v_{0 m} \rightarrow v_{0} \text { in } H .
\end{array}\right.
$$

Observe that (47) is a system of nonlinear differential equations where $h_{j m}$ are the unknowns. In fact, we have

$$
\left\{\begin{array}{l}
\left(\frac{w_{i}}{\sqrt{N}}, \frac{w_{j}}{\sqrt{N}}\right) h_{i m}^{\prime}(t)+\left(\mu a\left(t ; w_{i}, w_{j}\right)+\mu d\left(t ; w_{i}, w_{j}\right)+c\left(t ; w_{i}, w_{j}\right)\right) h_{i m}(t) \\
+b\left(t ; w_{i}, w_{l}, w_{j}\right) h_{i m}(t) h_{l m}(t)=\left(g, w_{j}\right) \quad j=1, \ldots, m \text { and } h_{j m}(0) w_{j}=v_{0 m}
\end{array}\right.
$$

Since $w_{i}$ are linearly independent, the matrix with the entries given by $\left(\frac{w_{i}}{\sqrt{N}}, \frac{w_{j}}{\sqrt{N}}\right)_{1 \leq i, j \leq n}$ is nonsingular. Then we can use the inverse of this matrix to obtain the nonlinear system

$$
\left\{\begin{array}{l}
h_{j m}^{\prime}(t)=\theta_{j i}(t)-\left(\alpha_{j i}(t)+\beta_{j i}(t)+\delta_{j i}(t)\right) h_{i m}(t)-\gamma_{k i k}(t) h_{i m}(t) h_{k m}(t) \\
h_{j m}(0)=i t h \text { component } v_{0 m} \quad j=1, \ldots, m
\end{array}\right.
$$

where $\alpha_{j i}(t), \beta_{j i}(t), \delta_{j i}(t), \gamma_{k i k}(t) \in \mathbb{R}$. From Carathcodory's theorem (sce Hale [4] pg 28), this system has a maximal solution defined in some interval $\left[0, t_{m}\right)$. If $t_{m}<T$, then $\left|h_{j m}\right|$ diverge to $+\infty$ as $t \rightarrow t_{m}$, but this cannot happen due to the first estimates that will be proved further. So $t_{m}=T$. Since the applications $t \rightarrow\left(g(t), w_{j}\right)$ belong to $L^{2}(0, T ; H)$, the same result holds to the functions $h_{j m}$, hence

$$
v_{m} \in L^{2}(0, T ; V) \text { and } v_{m}^{\prime} \in L^{2}(0, T ; V) \text {, }
$$

for any $m$. Now we will show the first estimates, that not depend on $m$, to the functions $v_{m}$. After that we will make the limit.

\section{First Estimates}


If we multiply (47) by $h_{j m}(t)$ and sum for $j=1, \ldots, m$, we have

$$
\begin{aligned}
& \left(\frac{v_{m}^{\prime}}{N}, v_{m}\right)+\mu a\left(t ; v_{m}, v_{m}\right)+b\left(t ; v_{m}, v_{m}, v_{m}\right)+c\left(t ; v_{m}, v_{m}\right)+\mu d\left(t ; v_{m}, v_{m}\right) \\
& =\left(g, v_{m}\right)
\end{aligned}
$$

From part (ii) of Lemma $4, b\left(t ; v_{m}, v_{m}, v_{m}\right)=0$ and, by (48)

$$
\left(\frac{v_{m}^{\prime}}{N}, v_{m}\right)=\frac{1}{2} \frac{d}{d t}\left|\frac{v_{m}(t)}{\sqrt{N}}\right|^{2}+\frac{1}{2} e\left(t ; v_{m}, v_{m}\right),
$$

so

$$
\begin{aligned}
& \frac{1}{2} \frac{d}{d t}\left|\frac{v_{m}(t)}{\sqrt{N}}\right|^{2}+\frac{1}{2} e\left(t ; v_{m}, v_{m}\right)+\mu a_{1}\left(t ; v_{m}, v_{m}\right)+\mu a_{2}\left(t ; v_{m}, v_{m}\right)+ \\
& c\left(t ; v_{m}, v_{m}\right)+\mu d\left(t ; v_{m}, v_{m}\right)=\left(g, v_{m}\right),
\end{aligned}
$$

hence

$$
\begin{aligned}
& \frac{1}{2} \frac{d}{d t}\left|\frac{v_{m}(t)}{\sqrt{N}}\right|^{2}+\mu a_{1}\left(t ; v_{m}, v_{m}\right)+\mu d\left(t ; v_{m}, v_{m}\right)=\left(g, v_{m}\right)- \\
& -\left[\frac{1}{2} e\left(t ; v_{m}, v_{m}\right)+\mu a_{2}\left(t ; v_{m}, v_{m}\right)+c\left(t ; v_{m}, v_{m}\right)\right] .
\end{aligned}
$$

Now observe that, from part (ii) of lemma 3 ,

$$
a_{0}\left\|v_{m}\right\|^{2} \leq a_{1}\left(t ; v_{m}, v_{m}\right),
$$

from part (iv) of lemma 4 and Young's inequality,

$$
\begin{gathered}
\left|c\left(t ; v_{m}, v_{m}\right)\right| \leq C\left\|v_{m}\right\|\left|\frac{v_{m}}{\sqrt{N}}\right| \leq \varepsilon\left\|v_{m}\right\|^{2}+C_{\varepsilon}\left|\frac{v_{m}}{\sqrt{N}}\right|^{2}, \\
\left\langle g, v_{m}\right\rangle \leq\|g\|_{H^{-1}}\left\|v_{m}\right\| \leq C_{\varepsilon}\|g\|_{H^{-1}}^{2}+\varepsilon\left\|v_{m}\right\|^{2}, \\
\left|a_{2}\left(t ; v_{m}, v_{m}\right)\right| \leq \int_{\Omega}\left|\beta_{l j}(t)\right|\left|\beta_{r j}(t)\right|\left|\frac{\partial v_{m i}}{\partial y_{r}}\right|\left|\frac{\partial N}{\partial y_{l}}\right|\left|\frac{v_{m i}}{N^{2}}\right| d y \\
\leq C \int_{\Omega}\left|\frac{\partial v_{m i}}{\partial y_{r}}\right|\left|\frac{\partial N}{\partial y_{l}}\right|\left|v_{m i}\right| d y \operatorname{using} \nabla N \in L^{2}\left(0, T ; L^{\infty}(\Omega)\right) \\
\leq C\|\nabla N\|_{L^{\infty}(\Omega)} \int_{\Omega}\left|\frac{\partial v_{m i}}{\partial y_{r}}\right|\left|v_{m i}\right| d y \leq C\|\nabla N\|_{L^{\infty}(\Omega)}\left\|v_{m}\right\|\left|v_{m}\right| \leq \\
\leq \varepsilon\left\|v_{m}\right\|^{2}+C_{\varepsilon}\|\nabla N\|_{L^{\infty}(\Omega)}^{2}\left|\frac{v_{m}}{\sqrt{N}}\right|^{2}
\end{gathered}
$$




$$
\begin{gathered}
\left|\frac{1}{2} e\left(t ; v_{m}, v_{m}\right)\right| \leq \int_{\Omega}\left|v_{m i}\right|\left|\frac{N^{\prime}}{N^{2}}\right|\left|v_{m i}\right| d y \leq \\
\leq C \int_{\Omega}\left|N^{\prime}\right|\left|v_{m}\right|^{2} d y \leq C \|\left. N^{\prime}\right|_{L^{\infty}(\Omega)}\left|\frac{v_{m}}{\sqrt{N}}\right|^{2} \\
\left|d\left(t ; v_{m}, v_{m}\right)\right| \leq \int_{\Omega} \frac{|G(N)|}{N}\left|v_{m}\right|\left|v_{m}\right| d y \leq C\left|\frac{v_{m}}{\sqrt{N}}\right|^{2} \\
\text { or }\left|d\left(t ; v_{m}, v_{m}\right)\right|=\left|\sqrt{\frac{G(N)}{N}} v_{m}\right|^{2} .
\end{gathered}
$$

Replacing the last six inequalities in (49), we get

$$
\begin{aligned}
& \frac{1}{2} \frac{d}{d t}\left|\frac{v_{m}(t)}{\sqrt{N}}\right|^{2}+\mu a_{0}\left\|v_{m}\right\|^{2}+\mu\left|\sqrt{\frac{G(N)}{N}} v_{m}\right|^{2} \leq C_{\varepsilon}\|g\|_{H^{-1}}^{2}+\varepsilon\left\|v_{m}\right\|^{2}+ \\
& +C\left\|N^{\prime}\right\|_{L^{\infty}(\Omega)}\left|\frac{v_{m}}{\sqrt{N}}\right|^{2}+\varepsilon\left\|v_{m}\right\|^{2}+C\|\nabla N\|_{L^{\infty}(\Omega)}^{2}\left|\frac{v_{m}}{\sqrt{N}}\right|^{2}+\varepsilon\left\|v_{m}\right\|^{2}+ \\
& +C_{\varepsilon}\left|\frac{v_{m}}{\sqrt{N}}\right|^{2} \\
& \frac{d}{d t}\left|\frac{v_{m}(t)}{\sqrt{N}}\right|^{2}+2\left(\mu a_{0}-3 \varepsilon\right)\left\|v_{m}\right\|^{2}+2 \mu\left|\sqrt{\frac{G(N)}{N}} v_{m}\right|^{2} \leq C_{\varepsilon}\|g\|_{H^{-1}}^{2}+ \\
& +C\left\|N^{\prime}\right\|_{L^{\infty}(\Omega)}\left|\frac{v_{m}}{\sqrt{N}}\right|^{2}+C\|\nabla N\|_{L^{\infty}(\Omega)}^{2}\left|\frac{v_{m}}{\sqrt{N}}\right|^{2}+C_{\varepsilon}\left|\frac{v_{m}}{\sqrt{N}}\right|^{2} .
\end{aligned}
$$

Take $\varepsilon>0$ such that $\mu a_{0}-3 \varepsilon>0$, then

$$
\begin{aligned}
& \frac{d}{d t}\left|\frac{v_{m}(t)}{\sqrt{N}}\right|^{2}+\left\|v_{m}\right\|^{2} \leq C\|g\|_{H^{-1}}^{2}+C\left\|N^{\prime}\right\|_{L^{\infty}(\Omega)}\left|\frac{v_{m}}{\sqrt{N}}\right|^{2} \\
& C\left(\|\nabla N\|_{L^{\infty}(\Omega)}^{2}+1\right)\left|\frac{v_{m}}{\sqrt{N}}\right|^{2}
\end{aligned}
$$

or

$$
\frac{d}{d t}\left|\frac{v_{m}(t)}{\sqrt{N}}\right|^{2}+\left\|v_{m}\right\|^{2} \leq C\|g\|_{I^{-1}}^{2}+\Phi(t)\left|\frac{v_{m}}{\sqrt{N}}\right|^{2},
$$

where $\Phi(t)=C\left\|N^{\prime}\right\|_{L^{\infty}(\Omega)}+C\left(\|\nabla N\|_{L^{\infty}(\Omega)}^{2}+1\right)$. Using Gronwall and observing that $\Phi$ is integrable in $[0, T]$, we get

$$
\left|\frac{v_{m}(t)}{\sqrt{N}}\right|^{2} \leq\left(\exp \int_{0}^{t} \Phi(s) d s\right)\left(\left|\frac{v_{m}(0)}{\sqrt{N(0)}}\right|^{2}\right)+\int_{0}^{t} C\|g\|_{H^{-1}}^{2} d s \leq C
$$


Hence $v_{m} \in L^{\infty}(0, T ; H)$. Integrating expression $(50)$ in $[0, t]$ with $t<T$ we have $v_{m}(t) \in L^{2}(0, T ; V)$, therefore

$\left(\frac{v_{m}(t)}{\sqrt{N}}\right)$ is uniformly bounded in $L^{\infty}\left(0, T ; L^{2}(\Omega)\right)$ and $v_{m}(t)$ is uniformly bounded in $L^{2}(0, T ; V)$

$$
\begin{aligned}
& \int_{0}^{t}\left|\nabla\left(\frac{v_{m}}{N}\right)\right|_{L^{2}}^{2} d t=\int_{0}^{t}\left|\frac{\nabla v_{m}}{N}+v_{m} \nabla\left(\frac{1}{N}\right)\right|_{L^{2}}^{2} d t \leq \\
& C+C \int_{0}^{t}|\nabla N|^{2} d t \text { then } \frac{v_{m}}{N} \in L^{2}\left(0, T ; H_{0}^{1}(\Omega)\right) .
\end{aligned}
$$

\section{Second Estimates}

Let $P_{m}: H \rightarrow V_{m}$ be the orthogonal projection of $H$ onto $V_{m}$, that is,

$$
P_{m} \varphi=\sum_{j=1}^{m}\left(\varphi, w_{j}\right) w_{j}
$$

Note that $P_{m} \in £\left(V_{s}, V_{s}\right)$. In fact, since $V_{s}$ is dense in $H$ and $V_{s} \hookrightarrow V \hookrightarrow H$, we can restrict $P_{m}$ to the space $V_{s}$ for our estimates. Consider the orthonormal basis $\left(w_{j}\right)$ and $\left(\frac{w_{j}}{\sqrt{\lambda_{j}}}\right)$ of $H$ and $V_{s}$, respectively. Then, using (46), we get

$$
\begin{aligned}
& \left\|P_{m}\right\|_{\mathcal{L}^{\prime}\left(V_{s}, V_{s}\right)}=\sup _{\|\varphi\|_{V_{s}} \leq 1}\left\|P_{m} \varphi\right\|_{V_{s}}=\sup _{\|\varphi\|_{V_{s}} \leq 1}\left\|\sum_{j=1}^{m}\left(\left(\varphi, \frac{w_{j}}{\sqrt{\lambda_{j}}}\right)\right)_{V_{s}} \frac{w_{j}}{\sqrt{\lambda_{j}}}\right\|_{V_{s}}= \\
& =\sup _{\|\varphi\|_{V_{s}} \leq 1} \sqrt{\sum_{j=1}^{m}\left|\left(\left(\varphi, \frac{w_{j}}{\sqrt{\lambda_{j}}}\right)\right)_{V_{s}}\right|^{2}} \leq \sup _{\|\varphi\|_{V_{s}} \leq 1} \sqrt{\sum_{j=1}^{\infty}\left|\left(\left(\varphi, \frac{w_{j}}{\sqrt{\lambda_{j}}}\right)\right)_{V_{s}}\right|^{2}}=1,
\end{aligned}
$$

therefore

$$
\left\|P_{m}\right\|_{\mathscr{L}\left(V_{s}, V_{s}\right)} \leq 1
$$

thus, by standard arguments:

$$
\left\|P_{m}^{*}\right\|_{\mathscr{L}\left(V_{s}^{\prime}, V_{s}^{\prime}\right)} \leq 1 .
$$

Observe that

$$
\begin{aligned}
& P_{m}\left(\frac{v_{m}}{N}\right)^{\prime}=\sum_{j=1}^{m}\left(\frac{v_{m}^{\prime}}{N}-v_{m} \frac{N^{\prime}}{N^{2}}, w_{j}\right) w_{j}=\sum_{j=1}^{m}\left(\sum_{i=1}^{m} \frac{h_{i m}^{\prime}}{N}\left(w_{i}, w_{j}\right) w_{j}-\right. \\
& \left.-\sum_{i=1}^{m} h_{i m} \frac{N^{\prime}}{N^{2}}\left(w_{i}, w_{j}\right)\right) w_{j}=\sum_{j=1}^{m} \frac{h_{j m}^{\prime}}{N} w_{j}-\sum_{j=1}^{m} h_{i m} \frac{N^{\prime}}{N^{2}} w_{j}=\frac{v_{m}^{\prime}}{N}-v_{m} \frac{N^{\prime}}{N}=\left(\frac{v_{m}}{N}\right)^{\prime}
\end{aligned}
$$


Hence, if we multiply the equation (47) by $w_{j}$ and sum for $j=1, \ldots, m$, we have

$$
\begin{aligned}
& \sum_{j=1}^{m}\left(\frac{v_{m}^{\prime}(t)}{N}, w_{j}\right) w_{j}+\mu \sum_{j=1}^{m} a\left(t ; v_{m}(t), w_{j}\right) w_{j}+\sum_{j=1}^{m} b\left(t ; v_{m}(t), v_{m}(t), w_{j}\right) w_{j}+ \\
& +\sum_{j=1}^{m} c\left(t ; v_{m}(t), w_{j}\right) w_{j}+\mu \sum_{j=1}^{m} d\left(t ; v_{m}(t), w_{j}\right) w_{j}=\sum_{j=1}^{m}\left(g, w_{j}\right) w_{j}
\end{aligned}
$$

Since

$$
\begin{aligned}
& \sum_{j=1}^{m}\left(\frac{v_{m}^{\prime}(t)}{N}, w_{j}\right) w_{j}=\sum_{j=1}^{m}\left(\left(\frac{v_{m}}{N}\right)^{\prime}+v_{m} \frac{N^{\prime}}{N^{2}}, w_{j}\right) w_{j}=P_{m}\left(\frac{v_{m}}{N}\right)^{\prime} \\
& +\sum_{j=1}^{m} e\left(t ; v_{m j}, w_{j}\right) w_{j}
\end{aligned}
$$

and using the notation of lemmas 2 and 3

$$
\begin{aligned}
& P_{m}\left(\frac{v_{m}}{N}\right)^{\prime}+<E(t) v_{m}, w_{j}>w_{j}+\sum_{j=1}^{m}\left(\mu<A(t) v_{m}, w_{j}>w_{j}+<B(t) v_{m}, w_{j}>w_{j}\right) \\
& +\sum_{j=1}^{m}\left(<C(t) v_{m}, w_{j}>w_{j}+\mu<D(t) v_{m}, w_{j}>w_{j}\right)=\sum_{j=1}^{m}\left(g, w_{j}\right) w_{j} ;
\end{aligned}
$$

hence, and from (52),

$$
\left(\frac{v_{m}}{N}\right)^{\prime}=\sum_{j=1}^{m}<g-(\mu A(t)+B(t)+C(t)+\mu D(t)+E(t)) v_{m}, w_{j}>w_{j},
$$

since $g-\mu A(t) v_{m}-B(t) v_{m}-C(t) v_{m}-\mu D(t) v_{m}-E(t) v_{m} \in V_{s}^{\prime}$, we have

$$
\left(\frac{v_{m}}{N}\right)^{\prime}=P_{m}^{*}\left(g-\mu A(t) v_{m}-B(t) v_{m}-C(t) v_{m}-\mu D(t) v_{m}-E(t) v_{m}\right) .
$$

Then taking the norm of $\left(\frac{v_{m}}{N}\right)^{\prime}$ in $V_{s}^{\prime}$, applying the triangular inequality, using $\left\|P_{m}^{*}\right\|_{f^{\prime}\left(V_{s}^{\prime}, V_{s}^{\prime}\right)} \leq 1$ and applying the Young's incquality repeatedly, it follows that

$$
\begin{aligned}
& \left\|\left(\frac{v_{m}}{N}\right)^{\prime}\right\|_{V_{s}^{\prime}(\Omega)}^{2} \leq 4\left(\left\|\mu A(t) v_{m}\right\|_{V_{s}^{\prime}(\Omega)}^{2}+\left\|B(t) v_{m}\right\|_{V_{s}^{\prime}(\Omega)}^{2}+\left\|C(t) v_{m}\right\|_{V_{s}^{\prime}(\Omega)}^{2}\right)+ \\
& +4\left(\left\|\mu D(t) v_{m}\right\|_{V_{s}^{\prime}(\Omega)}^{2}+\left\|E(t) v_{m}\right\|_{V_{s}^{\prime}(\Omega)}^{2}+\|g\|_{V_{s}^{\prime}(\Omega)}^{2}\right) .
\end{aligned}
$$

Now we estimate each term of the right hand side of the last expression:

- from part (iii) of lemma 2,

$$
\left\|\mu A(t) v_{m}\right\|_{V_{s}^{\prime}}=\sup _{\|w\|_{v_{s}} \leq 1}\left|<\mu A(t) v_{m}, w>\right| \leq C\left\|v_{m}\right\|_{V_{s}^{\prime}}\|w\|_{V_{s}} \leq C\left\|v_{m}\right\|_{V_{s}^{\prime}},
$$


and

$$
\int_{0}^{T}\left\|\mu A(t) v_{m}\right\|_{V_{s}^{\prime}(\Omega)}^{2} d s \leq C \int_{0}^{T}\left\|v_{m}\right\|_{V_{s}^{\prime}(\Omega)}^{2} d s \leq C \int_{0}^{T}\left\|v_{m}\right\|_{V(\Omega)}^{2} d s<\infty,
$$

since, from (51), $v_{m} \in L^{2}(0, T ; V)$ and $V \hookrightarrow V_{s}^{\prime}$.

- from part (iii) of lemma 4

$$
\left\|B(t) v_{m}\right\|_{V_{s}^{\prime}(\Omega)} \leq C\left\|v_{m}\right\|_{\left(L^{p}(\Omega)\right)^{n}}^{2}, \quad \text { wherc } \frac{1}{p}=\frac{1}{2}-\frac{1}{2 n} .
$$

Then

$$
\int_{0}^{T}\left\|B(t) v_{m}\right\|_{V_{s}^{\prime}(\Omega)}^{2} d s \leq \int_{0}^{T} C\left\|v_{m}\right\|_{\left(L^{p}(\Omega)\right)^{n}}^{4} d s .
$$

By (51) ; it holds that $v_{m} \in L^{2}(0, T ; V) \cap L^{\infty}(0, T ; H)$. Thus, from part (viii) of lemma $4, v_{m} \in L^{4}\left(0, T ;\left(L^{p}(\Omega)\right)^{n}\right)$. Hence and from the last incquality,

$$
\int_{0}^{T^{\prime}}\left\|B(t) v_{m}\right\|_{V_{s}^{\prime}(\Omega)}^{2} d s<\infty
$$

- from part (iv) of lemma 4

$$
\left\|C(t) v_{m}\right\|_{V_{s}^{\prime}(\Omega)} \leq\left|C(t) v_{m}\right| \leq C\left\|v_{m}\right\|_{V_{s}^{\prime}(\Omega)},
$$

that implies

$$
\int_{0}^{T}\left\|C(t) v_{m}\right\|_{V_{s}^{\prime}(\Omega)}^{2} d s \leq \int_{0}^{T} C\left\|v_{m}\right\|_{V_{s}^{\prime}(\Omega)}^{2} d s<\infty .
$$

- $\|g\|_{V_{s}^{\prime}(\Omega)} \leq\|g\|_{H(\Omega)}$.

Therefore

$$
\left(\frac{v_{m}}{N}\right)^{\prime} \text { is bounded in } L^{2}\left(0, T ; V_{s}^{\prime}(\Omega)\right) \text {. }
$$

Define

$$
W=\left\{v_{m} ; \frac{v_{m}}{N} \in L^{2}\left(0, T ; H_{0}^{1}(\Omega)\right) \text { and }\left(\frac{v_{m}}{N}\right)^{\prime} \in L^{2}\left(0, T ; V_{s}^{\prime}(\Omega)\right)\right\} ;
$$

with the norm $\left\|v_{m}\right\|_{L^{2}(0, T ; V(\Omega))}+\left\|v_{m}^{\prime}\right\|_{L^{2}\left(0, T ; V_{s}^{\prime}(\Omega)\right)}$. Since $V \hookrightarrow H$ and $H_{0}^{1}(\Omega) \hookrightarrow$ $L^{2}(\Omega) \hookrightarrow V_{s}^{\prime}(\Omega)$ compactly; by Aubim-Lions theorem $W \hookrightarrow L^{2}\left(0, T ; L^{2}(\Omega)\right)$ compactly. From this and by $(\check{\jmath} 1)$ there exists a subsequence of $\left(\frac{v_{m}}{N}\right)$, still denoted by $\left(\frac{v_{m}}{N}\right)$, and a function $v$ such that :

$$
v_{m} \rightarrow v \text { weakly in } L^{2}(0, T ; V)
$$




$$
\begin{gathered}
\frac{v_{m}}{N} \rightarrow \frac{v}{N} \text { weakly in } L^{2}\left(0, T ; H_{0}^{1}(\Omega)\right) \\
v_{m} \rightarrow^{*} v \text { weakly-star in } L^{\infty}(0, T ; H) \\
\frac{v_{m}}{N} \rightarrow^{*} \frac{v}{N} \text { weakly-star in } L^{\infty}\left(0, T ; L^{2}(\Omega)\right) \\
\left\{\begin{array}{c}
\frac{v_{m}}{N} \rightarrow \frac{v}{N} \text { strongly in } L^{2}\left(0, T ; L^{2}(\Omega)\right) \\
\left(\frac{v_{n_{i}}}{N} \rightarrow \frac{v}{N} \text { strongly in } L^{2}\left(0, T ; L^{2}(\Omega)\right)\right) \text { and a.e. in } Q \\
\left(\frac{v_{m}}{N}\right)^{\prime} \rightarrow\left(\frac{v}{N}\right)^{\prime} \text { wcakly in } L^{2}\left(0, T ; V_{s}^{\prime}(\Omega)\right)
\end{array}\right.
\end{gathered}
$$

Let $\Psi(t)$ be in $C_{0}^{\infty}(0, T)$; multiplying the equation (47) by $\Psi(t)$ and integrating with respect to $t$, we have

$$
\begin{aligned}
& \int_{0}^{T}\left(\left(\frac{v_{m}}{N}\right)^{\prime}, \Psi w_{j}\right) d t+\int_{0}^{T} \mu a\left(t ; v_{m}, \Psi w_{j}\right) d t+\int_{0}^{T} b\left(t ; v_{m}, v_{m}, \Psi w_{j}\right) d t+ \\
& \int_{0}^{T} c\left(t ; v_{m}, w_{j}\right) \Psi d t+\int_{0}^{T} \mu d\left(t ; v_{m}, \Psi w_{j}\right) d t+\int_{0}^{T} \mu e\left(t ; v_{m}, \Psi w_{j}\right) d t=\int_{0}^{T}\left(g, \Psi w_{j}\right) d t .
\end{aligned}
$$

Taking the limit for each term, by $(58)$

$$
\begin{gathered}
\int_{0}^{T}\left(\left(\frac{v_{m}}{N}\right)^{\prime}, \Psi w_{j}\right) d t=-\int_{0}^{T^{\prime}}\left(\frac{v_{m}}{N}, \Psi^{\prime} w_{j}\right) d t \rightarrow \int_{0}^{T}\left(\frac{v}{N}, \Psi^{\prime} w_{j}\right) d t \\
\int_{0}^{T^{\prime}} a\left(t ; v_{m}, \Psi w_{j}\right) d t=\int_{0}^{T} \int_{\Omega} \beta_{l j} \beta_{r j}\left(\frac{\partial v_{m i}}{\partial y_{r}}, \Psi \frac{\partial}{\partial y_{r}}\left(\frac{w_{j i}}{N}\right)\right) d y d t \rightarrow \int_{0}^{T^{\prime}} a\left(t ; v, \Psi w_{j}\right) d t
\end{gathered}
$$

by (56), due to lemma 3.2 TEMAN [10], pg 285 .

$$
\begin{gathered}
\int_{0}^{T} c\left(t ; v_{m}, w_{j}\right) \Psi d t=\int_{0}^{T} \int_{\Omega} \beta_{l r}^{\prime}(t) \alpha_{r j}(t) y_{j} \frac{\partial v_{m i}(y)}{\partial y_{l}} \frac{w_{j i}(y)}{N} \Psi d y d t \\
F(t)=\beta_{l r}^{\prime}(t) \alpha_{r j}(t) y_{j} \frac{w_{j i}(y)}{N} \in H_{0}^{1}(\Omega) \text { and } G_{m}(t)=\frac{\partial v_{m i}(y)}{\partial y_{l}} \in L^{2}(\Omega) \text { so } \\
\int_{0}^{T} c\left(t ; v_{m}, w_{j}\right) \Psi d t=\int_{0}^{T}\left(F(t), G_{m}(t)\right) d t=\int_{0}^{T}\left\langle F(t), G_{m}(t)\right\rangle d t
\end{gathered}
$$

converges (see pg 248 TEMAN [10]).

$$
\int_{0}^{T} d\left(t ; v_{m}, \Psi w_{j}\right) d t \rightarrow \int_{0}^{T} \int_{\Omega} G(N) v_{i}(y) \frac{w_{j i}(y)}{N} \Psi d y d t
$$


due to (59)

$$
\begin{gathered}
\int_{0}^{T} \mu e\left(t ; v_{m}, \Psi w_{j}\right) d t \rightarrow \int_{0}^{T} \int_{\Omega} v_{i}(y) \frac{N^{\prime}}{N^{2}} w_{j i}(y) \Psi d y d t \\
\text { since }\left|\int_{0}^{T} \int_{\Omega}\left(v_{m i}(y)-v_{i}(y)\right) \frac{N^{\prime}}{N^{2}} w_{j i}(y) \Psi d y d t\right| \rightarrow 0
\end{gathered}
$$

due to $(57)$ with the following statement: $\frac{N^{\prime}}{N^{2}} w_{j i}(y) \Psi \in L^{1}\left(0, T, L^{2}(\Omega)\right)$ since $\int_{0}^{T}\left\|\frac{N^{\prime}}{N^{2}} w_{j i}(y) \Psi\right\| d t \leq C \int_{0}^{T}\left\|N^{\prime}\right\|_{\infty}\left\|w_{j i}(y)\right\| d t<\infty$ from $N^{\prime} \in L^{1}\left(0, T, L^{\infty}(\Omega)\right)$.

Therefore, the theorem holds. The initial condition $v(0)=v_{0}$ is achieved from (55) and (60), that is, $v_{m} \rightarrow v$ wcakly in $L^{2}\left(0, T, V_{s}^{\prime}\right)$ and $v_{m}^{\prime} \rightarrow v$ wcakly- ${ }^{*}$ in $L^{2}\left(0, T, V_{s}^{\prime}\right)$ hence $v \in W^{1,2}\left(0, T, V_{s}^{\prime}\right) \Rightarrow u \in C\left(0, T, V_{s}^{\prime}\right)$ and $V_{s} \subset H \subset V_{s}^{\prime}$, $v \in V$ and $w \in H,\langle v, w\rangle_{V_{s} V_{S}^{\prime}}=(v, w)_{H}$.

\section{References}

[1] Adams, R., Sobolev Spaces, Academic Press, New York, 1975.

[2] Boldrini, J. L.; Lukaszczyk, J. P., Flow through Isotropic Granular (Non consolidated) Porous Media, Resenhas IME/USP, Vol. 3, No. 1 (1997), 2544.

[3] Brézis, H., Analyse fonctionelle - Théorie et applications, 2 tirage, Masson 1987.

[4] Hale, J. K., Ordinary Diferential Equation, Wilcy-Interscience, New York, 1969.

[5] Ladyzhenskaya, O. A., The Mathematical Theory of Viscous Incompressible Flow, Gordon and Breach, Ncw York, 1969.

[6] Lions, J. L., Quelques Méthodes de Résolution des Problèmes aux Limites non Linéaires, Dunod, Gauthier-Villars, Paris, 1969. 
[7] Miranda, M. M. \& Ferrel, J. L., The Navier-Stokes Equation in Noncylindrical Domain, Comput. Appl. Math., SBMAC, V. 16, n 3, (1997),247-265.

[8] Prieus Du Plessis, J. \& Masliyah, J. H., Flow through isotropic granular porous media, Transport in porous media, number 6, (1991), 207-221.

[9] Salvi, R., On the existence of weak solution of a nonlinear mixed problem for the Navier-Stokes equations in a time dependent domain, J. Fac. Sci. Lniv. Tokyo, Sec. IA, 32 (1985), 213-221.

[10] Teman, R., Navier-Stokes Equations - Theory and Numerical Analysis, North-Holland Publishing Company, Amesterdam, 1979.

\section{J. P. Lukaszczyk}

Departamento de Matemática

Universidade Federal de Santa Maria

97105-900 Santa Maria RS, Brasil

jpaulo@oslo.ccne.ufsm.com.br

L. P. Bonorino

Departamento de Matemática

Universidade Federal de Santa Maria

97105-900 Santa Maria RS, Brasil

lbonorin@zaz.com.br
C. A. Taschetto

Departamento de Matemática.

UNIFRA

Santa Maria RS, Brasil

carlost@unifra.br 